\title{
DNA-Organized Light-Harvesting Antennae: Energy Transfer in Polyaromatic Stacks Proceeds through Interposed Nucleobase Pairs
}

\author{
Caroline D. Böscha, Elif Abay ${ }^{a}$, Simon M. Langenegger ${ }^{a}$, Maryam Nazarib ${ }^{\mathrm{b}}$ Andrea Cannizzo ${ }^{\mathrm{b}}$, Thomas Feurer \\ and Robert Häner*,a \\ a Department of Chemistry and Biochemistry, University of Bern, Freiestrasse 3, CH-3012 Bern, Switzerland, E-mail: robert.haener@dcb.unibe.ch \\ ${ }^{\mathrm{b}}$ Institute for Applied Physics, University of Bern, Sidlerstrasse 5, 3012 Bern \\ Dedicated to Professor Philippe Renaud on the occasion of his $60^{\text {th }}$ birthday
}

\begin{abstract}
DNA-based light-harvesting antennae with varying arrangements of light-absorbing phenanthrene donor units and a pyrene acceptor dye were synthesized and tested for their light-harvesting properties. Excitation of phenanthrene is followed by rapid transfer of the excitation energy to the pyrene chromophore. A block of six light-absorbing phenanthrenes was separated from the site of the acceptor in a stepwise manner by an increasing number of intervening AT base pairs. Energy transfer occurs via interposed AT base pairs and is still detected when the phenanthrene antenna is separated by 5 AT base pairs.
\end{abstract}

Keywords: DNA • light-harvesting antenna $\bullet$ phenanthrene $\bullet$ pyrene $\bullet$ energy transfer

\section{Introduction}

DNA is a useful framework for the precise arrangement of chromophores and can, thus, serve in the development of novel types of nanomaterials. ${ }^{[1-}$ 9] Ordered arrays of chromophores are an essential requirement for the construction of artificial light-harvesting systems. In such systems, energy is absorbed by chromophores and subsequently transferred to an acceptor. The design and construction of efficient artificial systems is a continuing field of research. ${ }^{[10-23]}$ In previous work, we described various lightharvesting systems based on DNA-organized chromophores. ${ }^{[21,24,25]}$ In one approach, 3,6-dicarboxamide phenanthrene and 1,8-dicarboxamide pyrene were incorporated into DNA duplexes to build up light-harvesting antennae. ${ }^{[21]}$ Light is absorbed by a stack of phenanthrenes and subsequently transferred to a phenanthrene-pyrene exciplex. The fluorescence intensity showed to be proportional to the number of lightabsorbing phenanthrenes (up to eight phenanthrenes were used in a duplex). Another system describes the use of 1,8-dicarboxamide pyrene units and a cyanine dye (Cy5) in a DNA-assembled light-harvesting complexes (LHC). ${ }^{[24]}$ Duplexes with different numbers of pyrene and a $5^{\prime}$ terminally attached $C_{5} 5$ unit at one end were constructed. After excitation of pyrene, the excitation energy is transferred by FRET from pyrene excimers to the $\mathrm{Cy}_{5}$ acceptor. Furthermore, a DNA three-way junction was used for the arrangement of chromophores for the construction of lightharvesting antennae. ${ }^{[25]}$ A 3,6-dicarboxamide phenanthrene antenna is located in one of the three stems and an exchangeable acceptor is placed in proximity through the annealing of the third strand. Here, we present a DNA based light-harvesting antenna consisting of a pyrene acceptor and a stack of light-harvesting phenanthrene units, which is interrupted by a varying number of DNA base pairs (see Figure 1 for illustration). In contrast to previous projects 3,6-dialkynyl phenanthrene is used as the light- absorber as it has a higher absorption coefficient than the carboxamide derivative and was shown to stack more efficiently. ${ }^{[26]}$ A 1,8-dialkynyl pyrene was used as the acceptor dye. A series of duplexes was prepared in which the phenanthrene antenna is split in two parts by 1-5 A-T base pairs. The study allows addressing the question if excitation energy transfer along an array of phenanthrenes also takes place over a certain number of intervening base pairs.

\section{Results and Discussion}

DNA strands were synthesized by phosphoramidite chemistry using phenanthrene, pyrene and abasic site building blocks. Complementary DNA single strands were hybridized to yield duplexes with eight lightcollecting phenanthrene units and one pyrene acceptor (Table 1, duplexes 0-5). An abasic site analogue $(\varphi)$ was introduced opposite the pyrene building block to fix its positioning at the end of the chromophore stack. Only A-T base pairs were used in the vicinity of the chromophores, as the quenching effect of $G$ is well known. ${ }^{[27,28]}$ However, G-C base pairs were used at the ends to increase the stability of the duplexes. In duplex $\mathbf{0}$, the phenanthrenes form an uninterrupted stack, whereas in duplexes 1-5 the phenanthrenes are separated by one to five A-T base pairs. Additionally, two control duplexes were prepared, duplex Ref1 containing only the acceptor part (two phenanthrenes and one pyrene) and duplex Ref2 containing only a block of six continuous phenanthrenes. 

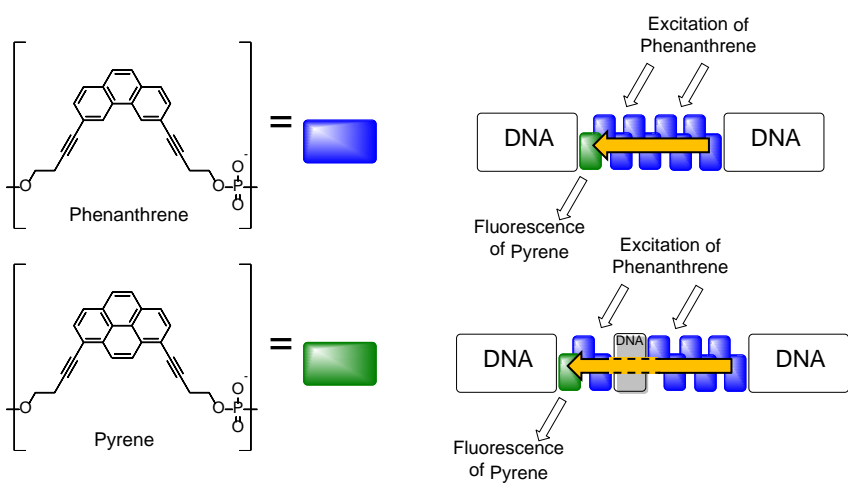

Figure 1. A) Structures of DNA-incorporated phenanthrene and pyrene building blocks; B) Schematic illustration of DNA based light-harvesting antennae without (top) and with (below) separating base pairs between phenanthrene units. Arrows indicate energy transfer.

Fluorescence spectra of the duplexes were recorded by exciting phenanthrene at $316 \mathrm{~nm}$ (Figure 2). All duplexes exhibit mainly pyrene emission with maxima at 403 and $425 \mathrm{~nm}$, indicating efficient energy transfer from phenanthrene to pyrene. The ratio of intensities of the two peaks varies within the series of duplexes. Duplexes 0-2 show higher intensity at $425 \mathrm{~nm}$ compared to $403 \mathrm{~nm}$, duplex 3 shows roughly the same intensities, whereas for duplexes 4 and 5 the emission at $403 \mathrm{~nm}$ is highest. This observation can be explained by different environments (next neighbors) for pyrene which can lead to different vibronic band intensities. A similar effect was observed for pyrene fluorescence in different solvents. ${ }^{[29]}$

In general, the duplexes with zero, one and two separating base pairs have about the same fluorescence intensities. If the stack of phenanthrene is separated by three base pairs the intensity decreases. Four or five base pairs lead to a further decrease of pyrene emission, whereas there is no significant difference between these two duplexes. At the same time, phenanthrene monomer emission around $380 \mathrm{~nm}$ increases if the phenanthrenes are separated by more than three base pairs (see Figure 2, inset). Therefore, energy transfer is not much affected by interruption of the phenanthrene stack by up to two base pairs. Further elongation of the gap, however, is followed by a decrease of energy transfer.

Table 1. Sequences of phenanthrene and pyrene modified DNA strands and structures of unnatural building blocks.

\begin{tabular}{|c|c|c|}
\hline Duplex & Oligo & Sequences \\
\hline \multirow{2}{*}{0} & o_a & $5^{\prime}$ GGC TAA $\varphi \alpha \alpha \alpha \alpha$ A TTA AAT CGC $3^{\prime}$ \\
\hline & o_b & $3^{\prime}$ CCG ATT Y $\alpha \alpha \alpha \alpha$ T AAT TTA GCG $5^{\prime}$ \\
\hline \multirow{2}{*}{1} & 1_a & $5^{\prime}$ GGC TAA $\varphi \alpha T^{\alpha} \alpha \alpha$ TTA AAT CGC $_{3}^{\prime}$ \\
\hline & 1_b & $3^{\prime}$ CCG ATT Y $\alpha$ A $\alpha \alpha \alpha$ AAT TTA GCG $5^{\prime}$ \\
\hline \multirow{2}{*}{2} & 2_a & $5^{\prime}$ GGC TAA $\varphi \alpha T^{\prime} A \alpha \alpha \alpha$ TA AAT CGC $3^{\prime}$ \\
\hline & 2_b & $3^{\prime}$ CCG ATT Y $\alpha$ A T $\alpha \alpha \alpha$ AT TTA GCG $5^{\prime}$ \\
\hline \multirow{2}{*}{3} & 3_a & $5^{\prime}$ GGC TAA $\varphi \alpha T^{A T} \alpha \alpha \alpha$ A AAT CGC $3^{\prime}$ \\
\hline & 3_b & $3^{\prime}$ CCG ATT Y $\alpha$ A TA $\alpha \alpha \alpha$ T TTA GCG $5^{\prime}$ \\
\hline
\end{tabular}

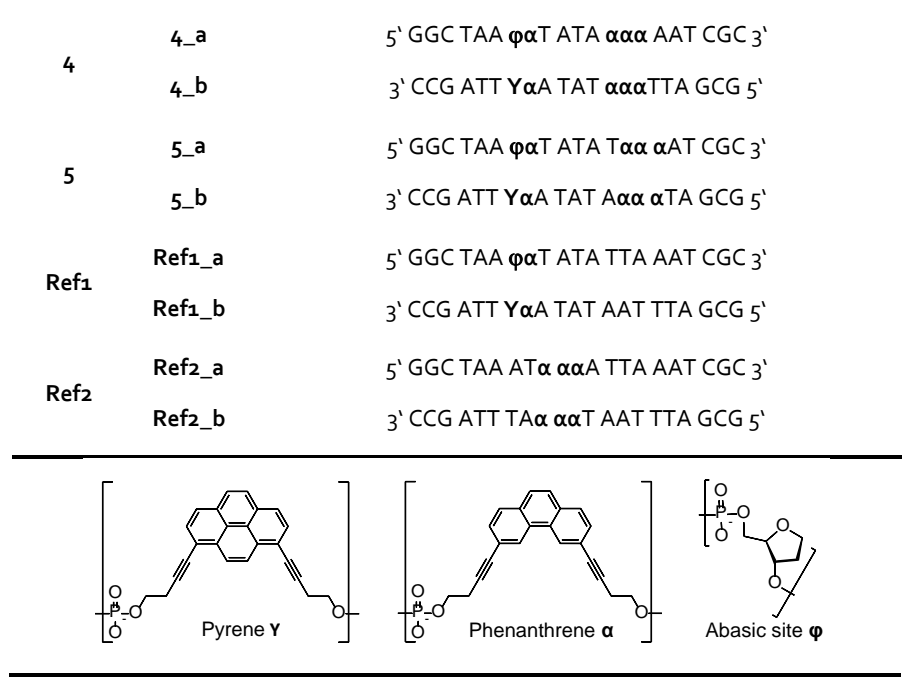

Quantum yields ( $\Phi_{F}$, Table 2 ) were measured for all single strands and duplexes by phenanthrene excitation at $316 \mathrm{~nm}$. Single strands containing only phenanthrene have values around $5 \%$, whereas single strands with pyrene have significant higher quantum yields $(29-46 \%)$, which again provides evidence for efficient excitation energy transfer from phenanthrene to pyrene. This increase in quantum yield cannot be explained by FRET theory. ${ }^{[30,31]}$ The observed efficiency rather suggests that excitation energy transfer occurs by quantum coherence mechanism. It is feasible that electronic coupling between the stacked phenanthrenes in the framework of the DNA duplex is strong enough to enable coherent energy transfer. ${ }^{[32-37]}$ This would also explain why the quantum yield of duplex $\mathbf{o}$ is lower than that of duplex $\mathbf{1}$. In duplex $\mathbf{o}$ the phenanthrene units are not separated by base pairs, therefore, electronic coupling should be strongest in this duplex. Very strong electronic coupling was shown to favor relaxation rather than energy transfer. ${ }^{\left[{ }^{[3]}\right.}$ Hence, energy transfer is optimal in duplex 1 and separation by additional base pairs results in reduced efficiency. Figure $3 \mathrm{~A}$ illustrates the measured (black) and calculated (blue) quantum yields of the duplexes. The deviation between the two values decreases with increasing number of separating base pairs. Using the quantum yields of duplexes Ref1 and Ref2 as a model for "infinite" separation of donor and acceptor, the theoretical quantum yield for no energy transfer was calculated to be $20 \%$ (see the Experimental Section). This is only slightly lower than the quantum yield of duplex $5\left(\Phi_{F}\right.$ $=24 \%$ ) and a value around $20 \%$ seems to be approached in the series with a growing number of intervening base pairs. Nevertheless, the measured quantum yield of duplex 5 is higher than the average of both single strands. Additionally, as can be seen in Figure 3 B, duplex 5 (red) shows a higher pyrene and lower phenanthrene fluorescence than the arithmetic sum of duplexes Ref1 and Ref2 (black, solid). This indicates that, although the phenanthrene units are separated by five base pairs, excitation energy transfer still takes place, albeit at a reduced level.

Table 2 also shows the melting temperatures (Tm) of all duplexes. Generally, the hybrids in which A-T base pairs separate the phenanthrene units (hybrids 1-5) are less stable compared to the hybrid in which phenanthrenes and pyrene form a continuous stack (hybrid o). The lowest 
Tm values were determined for duplexes 2 and 3 , in which the phenanthrenes are separated by two and three base pairs, respectively. All melting curves show no or only a negligible degree of hysteresis (see the Supplementary Material) indicating that duplex formation and disassembly are at thermodynamic equilibrium under experimental conditions. ${ }^{[39]}$

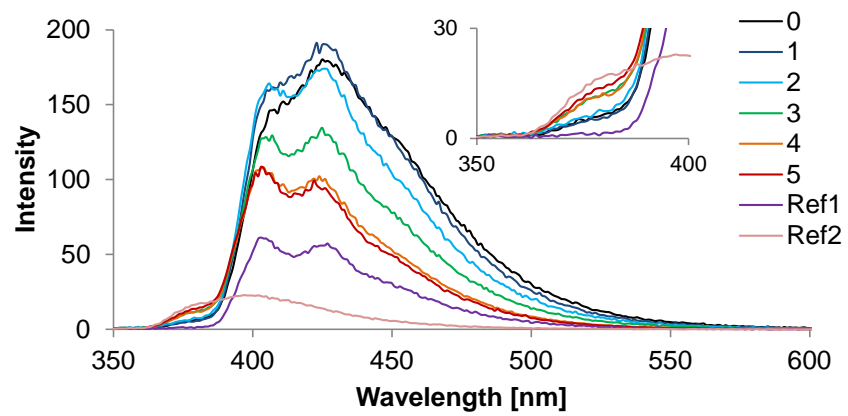

Figure 2. Fluorescence spectra of DNA-assembled light harvesting antennae.

Conditions: $0.25 \mu \mathrm{M}$ each strand, $10 \mathrm{mM}$ sodium phosphate buffer $\mathrm{pH} 7.0,100 \mathrm{mM}$ $\mathrm{NaCl}, \lambda_{\text {exc. }} 316 \mathrm{~nm}, 20^{\circ} \mathrm{C}$. Excitation slit: $2.5 \mathrm{~nm}$, emission slit: $5 \mathrm{~nm}$.

A)

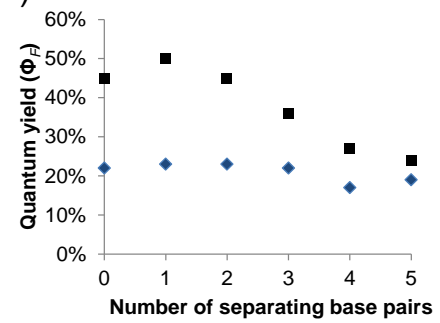

B)

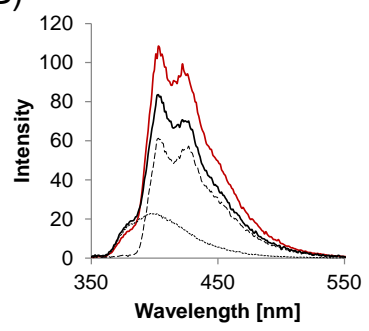

Figure 3. A) Quantum yields of duplexes $0-5$ (black) and the average quantum yield of the respective single strands $\mathbf{0} \_\mathbf{b}-\mathbf{5}$-b $\mathbf{b}$ (blue, see Table 2). B) Comparison of the measured fluorescence spectrum of duplex 5 (red) with the fluorescence spectra of Ref1 (dashed) and Ref2 (dotted) and the arithmetic sum of Ref1 and Ref2 (black). Conditions: see Figure 2.

Table 2. Quantum yields of modified single strands and duplexes (average value of single strands and measured) and melting temperatures $\left(T_{m}\right)$ of duplexes.

\begin{tabular}{|c|c|c|c|c|c|}
\hline Duplex & Oligo & $\begin{array}{c}\Phi_{\mathrm{F}} \text { (single } \\
\text { strands) }\end{array}$ & $\begin{array}{c}\Phi_{\mathrm{F}} \text { (avg. of single } \\
\text { strands) }\end{array}$ & $\begin{array}{c}\Phi_{\mathrm{F}} \\
\text { (duplexes) }\end{array}$ & $T_{m}$ \\
\hline \multirow{3}{*}{0} & o_a & $6 \%$ & \multirow{3}{*}{$22 \%$} & \multirow{3}{*}{$45 \%$} & \multirow{3}{*}{$51^{\circ} \mathrm{C}$} \\
\hline & & & & & \\
\hline & o_b & $37 \%$ & & & \\
\hline \multirow{3}{*}{1} & 1_a & $4 \%$ & \multirow{3}{*}{$23 \%$} & \multirow{3}{*}{$50 \%$} & \multirow{3}{*}{$49^{\circ} \mathrm{C}$} \\
\hline & & & & & \\
\hline & 1_b & $42 \%$ & & & \\
\hline \multirow{3}{*}{2} & 2_a & $5 \%$ & \multirow{3}{*}{$23 \%$} & \multirow{3}{*}{$45 \%$} & \multirow{3}{*}{$45^{\circ} \mathrm{C}$} \\
\hline & & & & & \\
\hline & 2_b & $41 \%$ & & & \\
\hline \multirow{3}{*}{3} & 3_a & $6 \%$ & \multirow{3}{*}{$22 \%$} & \multirow{3}{*}{$36 \%$} & \multirow{3}{*}{$45^{\circ} \mathrm{C}$} \\
\hline & & & & & \\
\hline & 3_b & $38 \%$ & & & \\
\hline
\end{tabular}

\begin{tabular}{|c|c|c|c|c|c|}
\hline \multirow{3}{*}{4} & $4 \_^{a}$ & $5 \%$ & \multirow{3}{*}{$17 \%$} & \multirow{3}{*}{$27 \%$} & \multirow{3}{*}{$46^{\circ} \mathrm{C}$} \\
\hline & & & & & \\
\hline & 4_b & $29 \%$ & & & \\
\hline \multirow{3}{*}{5} & 5_a & $4 \%$ & \multirow{3}{*}{$19 \%$} & \multirow{3}{*}{$24 \%$} & \multirow{3}{*}{$48^{\circ} \mathrm{C}$} \\
\hline & & & & & \\
\hline & 5_b & $33 \%$ & & & \\
\hline \multirow{3}{*}{ Ref1 } & Ref1_a & $6 \%$ & \multirow{3}{*}{$26 \%$} & \multirow{3}{*}{$56 \%$} & \multirow{3}{*}{$51^{\circ} \mathrm{C}$} \\
\hline & & & & & \\
\hline & Ref1_b & $46 \%$ & & & \\
\hline \multirow{3}{*}{ Ref2 $_{2}$} & Ref2_a & $5 \%$ & \multirow{3}{*}{$5 \%$} & \multirow{3}{*}{$8 \%$} & \multirow{3}{*}{$54^{\circ} \mathrm{C}$} \\
\hline & & & & & \\
\hline & Ref2_b & $5 \%$ & & & \\
\hline
\end{tabular}

a Calculated from the quantum yields of individual complementary strands.

\section{Conclusions}

In conclusion, a series of DNA-based light-harvesting antennae composed of light-collecting phenanthrenes and pyrene acceptors were synthesized and investigated. Excitation of phenanthrene leads to energy transfer to pyrene which then results in pyrene emission. Energy transfer is observed if the stack of phenanthrene units is interrupted by up to five DNA base pairs. The separation by one and two base pairs shows no decrease in energy transfer efficiency compared to a continuous phenanthrene stack. The light-harvesting antenna interrupted by one base pair has the highest efficiency of all tested duplexes. A separation by 3 to 5 base pairs leads to a gradual decrease in efficiency but energy transfer is still detected with 5 intervening base pairs. The data demonstrate that it is possible to assemble phenanthrene-based light-harvesting antennae which are mixed with natural DNA base pairs. This extends the range of potential designs and applications of DNA-based artificial light-harvesting complexes.

\section{Experimental Section}

\section{General Methods}

All reagents and solvents were purchased from commercial suppliers and used without further purification. Mass-spectrometric data were obtained on Thermo Fisher LTQ Orbitrap XL using Nano Electrospray lonization (NSI). UV-vis spectra were measured on a Cary 100 Bio spectrophotometer. Fluorescence and excitation spectra were measured on a Cary Eclipse spectrofluorimeter, excitation and emission slit widths were set to $5 \mathrm{~nm}$ if not mentioned otherwise.

\section{DNA Synthesis and Purification}


Syntheses of phenanthrene and pyrene phosphoramidites were described previously. ${ }^{[40,4]}$ The phosphoramidite derivative of building block $\varphi$, representing a stable analog of an abasic site, is commercially available (Glen Research). Modified DNA strands were assembled on an Applied Biosystems 394 DNA/RNA synthesizer. A standard cyanoethyl phosphoramidite coupling protocol was applied using a 3 -attached nucleoside-loaded controlled pore glass (CPG) support. After synthesis, the CPG-bound strands were cleaved and deprotected by treatment with $28-30 \% \mathrm{NH}_{4} \mathrm{OH}$ (aq) at $55^{\circ} \mathrm{C}$ overnight. The supernatants were collected. The solid supports were additionally washed three times with $1 \mathrm{ml}$ $\mathrm{EtOH} / \mathrm{H}_{2} \mathrm{O}$ 1:1. After combination and lyophilization of the supernatants, the crude oligomers were purified by reversed phase HPLC (Merck LiChroCART 250-4; LiChrospher 100, RP-18, $5 \mu \mathrm{m}$ ); aolvent A: $0.1 \mathrm{M}$ aqueous ammonium acetate; aolvent $\mathrm{B}: \mathrm{CH}_{3} \mathrm{CN}_{i} 1 \mathrm{ml} / \mathrm{min} ; \mathrm{T}=40^{\circ} \mathrm{C} ; \mathrm{B}[\%]$ $\left(t_{R}[\mathrm{~min}]\right)=\mathrm{O}(0) ; 5(2) ; 50(22)$. Identities of the oligomers were confirmed by ESI mass spectrometry (see the Supplementary Material). The samples were measured in negative ion mode in mixtures of water/acetonitrile/triethylamine. The purified oligomers were dissolved in $1 \mathrm{ml}$ Milli-O $\mathrm{H}_{2} \mathrm{O}$. Samples of the stock solutions were diluted and the absorbance at $326 \mathrm{~nm}$ was measured to determine the concentrations. The molar absorption coefficients of the oligomers were calculated using the $\varepsilon_{326}$ value of $35^{\prime} 400$ for phenanthrene.

\section{Quantum Yields}

The quantum yields (Table 2) were determined using quinine sulphate in $0.5 \mathrm{M} \mathrm{H}_{2} \mathrm{SO}_{4}$ as a standard according to the procedure described in the literature. ${ }^{[42]}$ The theoretical quantum yield for 'no energy transfer' was calculated assuming that the duplexes Ref1 and Ref2 do not interfere with each other using the following equations:

(eq.1) $\Phi_{\text {Ref }_{1}}=I_{\text {Ref }} / A_{\text {Ref1 }}$

(eq. 2) $\Phi_{\text {Ref }_{2}}=I_{\text {Ref }} / A_{\text {Ref } 2}$

(eq. 3) $\Phi_{\text {noET }}=\left(I_{\text {Ref }}+I_{\text {Ref }}\right) /\left(A_{R e f_{1}}+A_{\text {Ref }}\right)$

(eq. 4) $I_{\text {Ref1 }}=A_{\text {Ref }_{1}} * \Phi_{\text {Ref1 }_{1}} I_{\text {Ref } 2}=A_{R_{\text {ef2 }}} * \Phi_{\text {Ref }_{2}}$

(eq. 5) $\Phi_{\text {no ET }}=\left(A_{\text {Ref }_{1}} * \Phi_{\text {Ref }_{1}}\right) /\left(A_{\text {Ref }_{1}}+A_{\text {Ref }_{2}}\right)+\left(A_{\text {Ref }_{2}} * \Phi_{\text {Ref }_{2}}\right) /\left(A_{\text {Ref }_{1}}+A_{\text {Ref }_{2}}\right)$

$A_{\text {Ref }}=2$ and $A_{\text {Ref2 }}=6$ (respective number of phenanthrenes)

(eq. 6) $\Phi_{\text {no ET }}=\Phi_{\text {Refi } 1 / 4}+3 * \Phi_{\text {Ref } 2} / 4=20 \%$

\section{Supplementary Material}

Supporting information for this article is available on the WWW under http://dx.doi.org/10.1002/MS-number.

\section{Acknowledgements}

This work was supported by the Swiss National Foundation (Grant 200020-169030 and NCCR MUST).

\section{Author Contribution Statement}

C.D.B, E.A. and M.N. performed the experiments and analyzed the data. C.D.B, S.M.L., A.C., T.F. and R.H. conceived and designed the experiments, and wrote the paper.

\section{References}

[1] DNA in Supramolecular Chemistry and Nanotechnology, Eds.: E. Stulz, G. H. Clever, John Wiley \& Sons, Chichester 2015

[2] Y. N. Teo, E. T. Kool, 'DNA-Multichromophore Systems', Chem.Rev. 2012, 112, 4221-4245.

[3] R. Varghese, H. A. Wagenknecht, 'DNA as a supramolecular framework for the helical arrangements of chromophores: towards photoactive DNA-based nanomaterials', Chem.Commun. 2009, 2615-2624.

[4] V. L. Malinovskii, D. Wenger, R. Häner, 'Nucleic acid-guided assembly of aromatic chromophores', Chem.Soc.Rev. 2010, 39, 410-422.

[5] E. Stulz, 'Nanoarchitectonics with Porphyrin Functionalized DNA', Acc.Chem.Res. 2017, 50, 823-831.

[6] F. A. Aldaye, A. L. Palmer, H. F. Sleiman, 'Assembling materials with DNA as the guide', Science 2008, 321, 1795-1799.

[7] N. C. Seeman, 'Nanomaterials Based on DNA', Annu.Rev.Biochem. 2010, 79, 65-87.

[8] A. V. Pinheiro, D. R. Han, W. M. Shih, H. Yan, 'Challenges and opportunities for structural DNA nanotechnology', Nature Nanotechnology 2011, 6, 763-772.

[9] W. Pfeifer, B. Saccá, 'From Nano to Macro through Hierarchical Self-Assembly: The DNA Paradigm', Chembiochem 2016, 17, 10631080

[10] D. Gust, T. A. Moore, A. L. Moore, 'Mimicking photosynthetic solar energy transduction', Acc.Chem.Res. 2001, 34, 40-48.

[11] M. S. Choi, T. Yamazaki, I. Yamazaki, T. Aida, 'Bioinspired molecular design of light-harvesting multiporphyrin arrays', Angew. Chem.Int.Ed. 2004, 43, 150-158.

[12] H. Yeo, K. Tanaka, Y. Chujo, 'Construction and properties of a light-harvesting antenna system for phosphorescent materials based on oligofluorene-tethered Pt-porphyrins', RSC Adv. 2017, 7, 10869-10874

[13] X. Feng, X. Ding, L. Chen, Y. Wu, L. Liu, M. Addicoat, S. Irle, Y. Dong, D. Jiang, 'Two-dimensional artificial light-harvesting antennae with predesigned high-order structure and robust photosensitising activity', Sci.Rep. 2016, 6, 32944.

[14] A. Uetomo, M. Kozaki, S. Suzuki, K. i. Yamanaka, O. Ito, K. Okada, 'Efficient Light-Harvesting Antenna with a Multi-Porphyrin Cascade', J.Am.Chem.Soc. 2011, 133, 13276-13279.

[15] R. K. Dubey, D. Inan, S. Sengupta, E. J. R. Sudh+Âlter, F. C. Grozema, W. F. Jager, 'Tunable and highly efficient lightharvesting antenna systems based on 1,7-perylene-3,4,9,10tetracarboxylic acid derivatives', Chem.Sci. 2016, 7, 3517-3532.

[16] C. F. Calver, K. S. Schanze, G. Cosa, 'Biomimetic Light-Harvesting Antenna Based on the Self-Assembly of Conjugated Polyelectrolytes Embedded within Lipid Membranes', Acs Nano 2016, 10, 10598-10605.

[17] J.-J. Li, Y. Chen, J. Yu, N. Cheng, Y. Liu, 'A Supramolecular Artificial Light-Harvesting System with an Ultrahigh Antenna Effect', Adv.Mater. 2017, 29, 1701905.

[18] W. P. Klein, S. A. Diaz, S. Buckhout-White, J. S. Melinger, P. D. Cunningham, E. R. Goldman, M. G. Ancona, W. Kuang, I. L. Medintz, 'Utilizing HomoFRET to Extend DNA-Scaffolded Photonic Networks and Increase Light-Harvesting Capability', Adv.Opt.Mater. 2017, 6, 1700679

[19] J. G. Woller, J. K. Hannestad, B. Albinsson, 'Self-Assembled Nanoscale DNA-Porphyrin Complex for Artificial Light Harvesting', J.Am.Chem.Soc 2013, 135, 2759-2768.

[20] P. K. Dutta, R. Varghese, J. Nangreave, S. Lin, H. Yan, Y. Liu, 'DNA-Directed Artificial Light-Harvesting Antenna', J.Am.Chem.Soc. 2011, 133, 11985-11993. 
[21] F. Garo, R. Häner, 'A DNA-Based Light-Harvesting Antenna', Angew.Chem.Int.Ed. 2012, 51, 916-919.

[22] E. Boulais, N. P. D. Sawaya, R. Veneziano, A. Andreoni, J. L. Banal, T. Kondo, S. Mandal, S. Lin, G. S. Schlau-Cohen, N. W. Woodbury, H. Yan, A. Aspuru-Guzik, M. Bathe, 'Programmed coherent coupling in a synthetic DNA-based excitonic circuit', Nat.Mater. 2017, 17, 159-166.

[23] Y. Choi, L. Kotthoff, L. Olejko, U. Resch-Genger, I. Bald, 'DNA Origami-Based Förster Resonance Energy-Transfer Nanoarrays and Their Application as Ratiometric Sensors', ACS Appl.Mater.Interfaces 2018, 10, 23295-23302.

[24] O. O. Adeyemi, V. L. Malinovskii, S. M. Biner, G. Calzaferri, R. Häner, 'Photon harvesting by excimer-forming multichromophores', Chem.Commun. 2012, 48, 9589-9591.

[25] M. Probst, S. M. Langenegger, R. Häner, 'A modular LHC built on the DNA three-way junction', Chem.Commun. 2014, 50, 159-161.

[26] C. B. Winiger, S. Li, G. R. Kumar, S. M. Langenegger, R. Häner, 'Long-Distance Electronic Energy Transfer in Light-Harvesting Supramolecular Polymers', Angew. Chem.Int.Ed. 2014, 53, 1360913613 .

[27] S. O. Kelley, J. K. Barton, 'Electron Transfer Between Bases in Double Helical DNA', Science 1999, 283, 375-381.

[28] F. Garo, R. Häner, 'Influence of a GC Base Pair on Excitation Energy Transfer in DNA-Assembled Phenanthrene $\pi$-Stacks', Bioconjug.Chem. 2012, 23, 2105-2113.

[29] K. Kalyanasundaram, J. K. Thomas, 'Environmental effects on vibronic band intensities in pyrene monomer fluorescence and their application in studies of micellar systems', J.Am.Chem.Soc 1977, 99, 2039-2044.

[30] T. Förster, 'Zwischenmolekulare Energiewanderung und Fluoreszenz', Ann.Phys. 1948, 2, 55-75.

[31] C. Berney, G. Danuser, 'FRET or No FRET: A Quantitative Comparison', Biophys.J. 2003, 84, 3992-4010.

[32] E. Collini, 'Spectroscopic signatures of quantum-coherent energy transfer', Chem.Soc.Rev. 2013, 42, 4932-4947.

[33] E. Collini, G. D. Scholes, 'Coherent Intrachain Energy Migration in a Conjugated Polymer at Room Temperature', Science 2009, 323, 369-373.

[34] J. Struempfer, M. Sener, K. Schulten, 'How Quantum Coherence Assists Photosynthetic Light-Harvesting', J.Phys.Chem.Lett. 2012, 3, 536-542.

[35] G. S. Engel, T. R. Calhoun, E. L. Read, T. K. Ahn, T. Mancal, Y. C. Cheng, R. E. Blankenship, G. R. Fleming, 'Evidence for wavelike energy transfer through quantum coherence in photosynthetic systems', Nature 2007, 446, 782-786.

[36] G. R. Fleming, G. D. Scholes, 'Physical chemistry - Quantum mechanics for plants', Nature 2004, 431, 256-257.

[37] I. Hwang, G. D. Scholes, 'Electronic Energy Transfer and Quantum-Coherence in pi-Conjugated Polymers', Chem.Mater. 2011, 23, 610-620.

[38] A. Chenu, G. D. Scholes, 'Coherence in Energy Transfer and Photosynthesis', Annu.Rev. Phys. Chem. 2015, 66, 69-96.

[39] R. A. J. Darby, M. Sollogoub, C. McKeen, L. Brown, A. Risitano, N. Brown, C. Barton, T. Brown, K. R. Fox, 'High throughput measurement of duplex, triplex and quadruplex melting curves using molecular beacons and a LightCycler', Nucl.Acids. Res. 2002, 30.

[40] H. Bittermann, D. Siegemund, V. L. Malinovskii, R. Häner, 'Dialkynylpyrenes: strongly fluorescent, environment-sensitive DNA building blocks', J.Am.Chem.Soc. 2008, 130, 15285-15287.

[41] C. B. Winiger, S. M. Langenegger, G. Calzaferri, R. Häner, 'Formation of Two Homo-chromophoric H-Aggregates in DNAAssembled Alternating Dye Stacks', Angew. Chem.Int.Ed. 2015, 54, 3643-3647.

[42] S. Fery-Forgues, D. Lavabre, 'Are Fluorescence Quantum Yields So Tricky to Measure? A Demonstration Using Familiar Stationery Products', J.Chem.Educ. 1999, 76, 1260. 


\section{Entry for the Table of Contents}

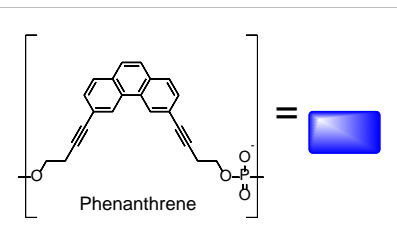
Excitation of Phenanthrene


Fluorescence
of Pyrene 